\title{
Taxocenose de anuros de uma mata semidecídua do interior do Estado de São Paulo e comparações com outras taxocenoses do Estado, sudeste do Brasil
}

\author{
Juliana Zina ${ }^{1,3}$, João Ennser ${ }^{l}$, Sarah Cristina Piacentini Pinheiro', \\ Célio Fernando Baptista Haddad ${ }^{I}$ Luís Felipe de Toledo ${ }^{I}$
}

Biota Neotropica $v 7(n 2)$ - http://www.biotaneotropica.org.br/v7n2/pt/abstract?article + bn00607022007

\author{
Recebido em 03/07/06 \\ Versão reformulada recebida em 08/02/07 \\ Publicado em 01/05/07 \\ ${ }^{1}$ Departamento de Zoologia, Instituto de Biociências, Universidade Estadual Paulista - UNESP, \\ Av. 24 A, n. 1515, CEP 13506-900, Bela Vista, Rio Claro, SP, Brasil \\ ${ }^{2}$ Departamento de Ciências Biológicas, Escola Superior de Agricultura “Luiz de Queiroz”, \\ Universidade de São Paulo, Av. Pádua Dias, n. 11, CP 9, CEP 13418-900 Piracicaba, SP, Brasil, \\ http://www.esalq.usp.br/ \\ ${ }^{3}$ Autor para correspondência: Juliana Zina, e-mail: juzina@ rc.unesp.br
}

\begin{abstract}
Zina, J., Ennser, J., Pinheiro, S.C.P., Haddad, C.F.B. \& Toledo, L.F. Anurans of a semidecidual forest in the interior of the São Paulo state and comparisons with other assemblages of the state, southeastern Brazil. Biota Neotrop. May/Aug 2007 vol. 7, no. 2 http://www.biotaneotropica.org.br/v7n2/pt/abstract?article+bn00607022007. ISSN 1676-0603.

The present study was conducted in a remnant of semi-deciduous forest, Mata São José (MSJ), and in its adjacent areas in the municipality of Rio Claro, state of São Paulo, Brazil. Information about anuran species richness and their spatial and temporal distribution were collected from August 2002 to July 2003. Most of the 24 registered species called and breed in open areas around the remnant. Only three species, Hypsiboas lundii, $H$. prasinus, and Proceratophrys boiei, used environments inside the fragment as reproductive sites and only $H$. prasinus used also environments around the remnant. The majority of the species were found during the rainy season and temporary environments were the most used for reproduction. The species composition of MSJ was compared with 10 other localities with the following vegetation composition: a preserved tropical rain forest, ecotone of tropical rain forest and semi-deciduous altered forest, preserved Cerrado, and disturbed areas (used as agriculture and pasture for cattle). The anuran composition of the MSJ and that from other semi-deciduous forest were more similar to the anuran composition of more perturbed areas and to the anuran composition of Cerrado then to the anuran composition of the rain forest habitats. There was no significant correlation between the distance of the areas and the similarity ratio of them. Proceratophrys boiei and H. lundii appear to be the species more affected by habitat fragmentation in the state of São Paulo due to their reproductive ecology and dependence of forested habitats.

Keywords: fragmentation, spatial and temporal distribution, anuran species composition.

Resumo

Zina, J., Ennser, J., Pinheiro, S.C.P., Haddad, C.F.B. \& Toledo, L.F. Taxocenose de anuros de uma mata semidecídua do interior do Estado de São Paulo e comparações com outras taxocenoses do Estado, sudeste do Brasil. Biota Neotrop. May/Aug 2007 vol. 7, no. 2 http://www.biotaneotropica.org.br/v7n2/pt/ abstract?article+bn00607022007. ISSN 1676-0603.

O presente estudo foi realizado em um fragmento de floresta semidecídua, Mata São José (MSJ), e em áreas adjacentes ao fragmento localizado no município de Rio Claro, Estado de São Paulo, Brasil. Informações sobre a composição e distribuição espacial e temporal de espécies de anuros foram coletadas entre agosto de 2002 e julho de 2003. Foram registradas 24 espécies, sendo que a maioria foi observada em atividade de vocalização e reprodução nas áreas abertas adjacentes ao fragmento. Apenas três espécies, Hypsiboas lundii, H. prasinus e Proceratophrys boiei foram observadas utilizando ambientes no interior do fragmento para a reprodução e apenas $H$. prasinus foi registrada também em ambientes adjacentes ao fragmento. A maioria das espécies foi registrada durante a estação chuvosa da região e os ambientes temporários foram os mais utilizados para reprodução. A composição de espécies da MSJ foi comparada com a de outras 10 localidades com as seguintes fisionomias vegetais e status de conservação: floresta tropical preservada, ecótono de floresta ombrófila e floresta estacional semidecídua pouco preservada, Cerrado preservado e áreas antropizadas principalmente pela implantação de agricultura e pastagem. A composição de espécies da MSJ e da área de floresta estacional semidecídua foram mais similares entre si e com áreas perturbadas e área de Cerrado que quando comparadas a composição de espécies da área de floresta tropical preservada. Não houve correlação entre as distâncias e a similaridade na composição da anurofauna das localidades comparadas. Proceratophrys boiei e H. lundii aparentemente são as espécies mais afetadas pelo processo de fragmentação ambiental no Estado de São Paulo, provavelmente devido a sua ecologia e dependência de ambientes mais florestados.
\end{abstract}


Palavras-chave: fragmentação, distribuição espacial e temporal, composição de espécies.

\section{Introdução}

As regiões tropicais abrigam a maior diversidade de espécies de anfíbios anuros do mundo (Heyer et al. 1990, Duellman 1999). Embora o número de estudos sobre a anurofauna brasileira tenha aumentado consideravelmente nos últimos anos (Arzabe 1999, Bernarde \& Machado 2001, Bertoluci \& Rodrigues 2001, Toledo et al. 2003, Ávila \& Ferreira 2004), a diversidade de anfíbios anuros neotropicais ainda é pouco conhecida, especialmente no Estado de São Paulo, que abriga cerca de $35 \%$ das espécies conhecidas de anuros no país e cerca de 5\% da diversidade mundial (veja Haddad 1998). Esta elevada riqueza em espécies de anuros no Estado de São Paulo é provavelmente resultado da extensa faixa florestal, genericamente conhecida como Mata Atlântica, que originalmente cobria o Estado, bem como da existência de uma ampla gama de outros ecossistemas (Haddad 1998). A escassez de estudos é ainda mais problemática quando consideramos áreas afetadas por ações antrópicas, como desmatamentos e poluição ambiental (Silvano et al. 2003), as quais, comprovadamente, alteram as taxocenoses e eliminam populações de anuros que, muitas vezes, sequer foram estudadas (Young et al. 2000).

A fragmentação de hábitats e os desmatamentos são tidos como os principais responsáveis pela perda de diversidade e extinções locais de espécies de anuros em todo o mundo (Hitchings \& Beebee 1997, Johnston \& Frid 2002). Além dos efeitos diretos (perda de hábitat), a fragmentação prejudica a dispersão das espécies de anuros, levando a uma diminuição da abundância populacional e da riqueza de espécies em fragmentos isolados (Funk et al. 2005) e permitindo que espécies generalistas ampliem suas distribuições podendo substituir populações nativas mais especializadas (Kats \& Ferrer 2003).

No Estado de São Paulo, os desmatamentos ocorreram de forma drástica e rápida, de modo que hoje restam apenas cerca de $7 \%$ da sua cobertura vegetal original (Viana \& Tabanez 1996). As florestas estacionais semideciduais foram as mais afetadas, sendo que esta fisionomia, atualmente, restringe-se a apenas $2 \%$ do que antes representava no Estado (Viana \& Tabanez 1996), grande parte reduzida a pequenos fragmentos próximos a centros urbanos.

O presente estudo tem como objetivos (i) obter informações sobre a composição e a distribuição espacial de espécies de anuros em um fragmento de floresta estacional semidecídua e em áreas alteradas por ação antrópica no seu entorno, (ii) determinar os padrões reprodutivos apresentados pelas espécies encontradas e verificar quais mecanismos de segregação que podem estar atuando nessa taxocenose, e (iii) comparar a composição da taxocenose desse fragmento de floresta com taxocenoses de anuros de áreas preservadas de Mata Atlântica, de áreas preservadas de Cerrado e de áreas alteradas por ação humana no Estado de São Paulo.

\section{Material e Métodos}

\section{1. Área de estudo}

Este estudo foi desenvolvido em um fragmento de uma Reserva Legal (Mata São José: MSJ) $\left(22^{\circ} 22^{\prime} \mathrm{S}\right.$ e $47^{\circ} 28^{\prime} \mathrm{W} ; 650 \mathrm{~m}$ de altitude) e em corpos d'água vizinhos ao fragmento localizados em matriz de cana de açúcar no Município de Rio Claro, Estado de São Paulo. A Mata São José (MSJ) possui 243 ha de floresta estacional semidecídua montana (sensu Veloso \& Góes-Filho 1982) e o clima da região é do tipo Cwa, segundo a classificação de Köeppen, com verões quentes e úmidos e invernos frios e secos

\section{Coleta de dados}

Dois diferentes tipos de corpos d'água no interior do fragmento e dois no seu entorno foram visitados semanalmente, durante a estação chuvosa e quinzenalmente, durante a estação seca, entre agosto de 2002 e julho de 2003 . No interior do fragmento foram amostrados: um córrego com cerca de $30 \mathrm{~cm}$ de profundidade e $1 \mathrm{~m}$ de largura com solo arenoso e trechos de leito pedregoso e três poças temporárias com dimensões que variaram de 3 a $5 \mathrm{~m}$ de largura e $20 \mathrm{~cm}$ de profundidade máxima. No entorno do fragmento foram estudados os seguintes ambientes: lagoa permanente de aproximadamente $10 \mathrm{~m}$ de diâmetro e $2 \mathrm{~m}$ de profundidade máxima e cinco poças temporárias formadas em meio ao canavial, com diâmetro variando entre 2 e 10 m e profundidade máxima de $10 \mathrm{~cm}$.

As observações naturalísticas eram iniciadas pouco antes do ocaso e encerradas por volta de 24:00 horas. Durante as observações foram registrados o número de espécies em atividade de vocalização, o tipo de ambiente (área florestada ou área aberta) e de corpo de água (lagoa permanente, poça temporária e córrego de água corrente). O período reprodutivo das espécies foi determinado pela presença de machos em atividade de vocalização ou pela presença de girinos em estágio inicial de desenvolvimento. Espécimes testemunho foram depositados na coleção de anuros CFBH, do Departamento de Zoologia, Instituto de Biociências, Universidade Estadual Paulista, Rio Claro, São Paulo.

\section{Análise dos dados}

Dados climatológicos (média mensal da temperatura do ar e pluviosidade mensal total) foram obtidos na Estação Meteorológica da UNESP de Rio Claro (CEAPLA), distante cerca de $8 \mathrm{~km}$ do local estudado. Para verificar a existência de relação entre o número de espécies em atividade de vocalização e os dados climatológicos foi realizada uma análise de regressão, sendo considerados significativos valores de $\mathrm{P}<0,05$ (Zar 1999).

A composição de espécies da área estudada foi comparada com a de outras 10 localidades do Estado de São Paulo (Figura 1):

1) Município de Guararapes ( $21^{\circ} 16^{\prime} \mathrm{S}$ e $50^{\circ} 37^{\prime} \mathrm{W} ; 400 \mathrm{~m}$ de altitude): área modificada por atividade humana cuja vegetação nativa era caracterizada por Cerrado e floresta estacional semidecídua. O levantamento da anurofauna desta localidade foi realizado por Bernarde \& Kokubum (1999) entre 1996 e 1998, quando foram visitados três açudes permanentes, poças temporárias e brejos. Os ambientes visitados pelos autores localizam-se em área preservada e áreas antropizadas. A distância de Guararapes à MSJ é de aproximadamente $365 \mathrm{~km}$;

2) Distrito de Nova Itapirema (21 $11^{\prime} \mathrm{S}$ e $49^{\circ} 42^{\prime} \mathrm{W}$; $460 \mathrm{~m}$ de altitude), município de Nova Aliança: sua fisionomia original era de floresta estacional semidecídua e Cerrado, hoje é uma área degradada com fortes influências antrópicas. O levantamento da anurofauna desta localidade foi realizado por Rossa-Feres \& Jim (2001) entre 1992 e 1995 em um açude temporário escavado em uma área de pastagem. Dista cerca de $270 \mathrm{~km}$ da MSJ;

3) Estação Ecológica de Itirapina (EEI: $22^{\circ} 15^{\prime}$ S e $47^{\circ} 49^{\prime} \mathrm{W}$; $750 \mathrm{~m}$ de altitude), município de Itirapina: representa um dos últimos remanescentes de Cerrado no Estado de São Paulo. O levantamento da anurofauna foi realizado por Brasileiro et al. (2005), entre 1999 e 2002, quando foram visitadas poças permanentes e temporárias, lagoas temporárias, córregos, brejos e represas em diferentes tipos vegetacionais de Cerrado preservado. Dista cerca de $40 \mathrm{~km}$ da Mata São José;

4) Floresta Estadual Edmundo Navarro de Andrade (FEENA: $22^{\circ} 22^{\prime} \mathrm{S}$ e $47^{\circ} 28^{\prime} \mathrm{W}$; $650 \mathrm{~m}$ de altitude), município de Rio Cla- 


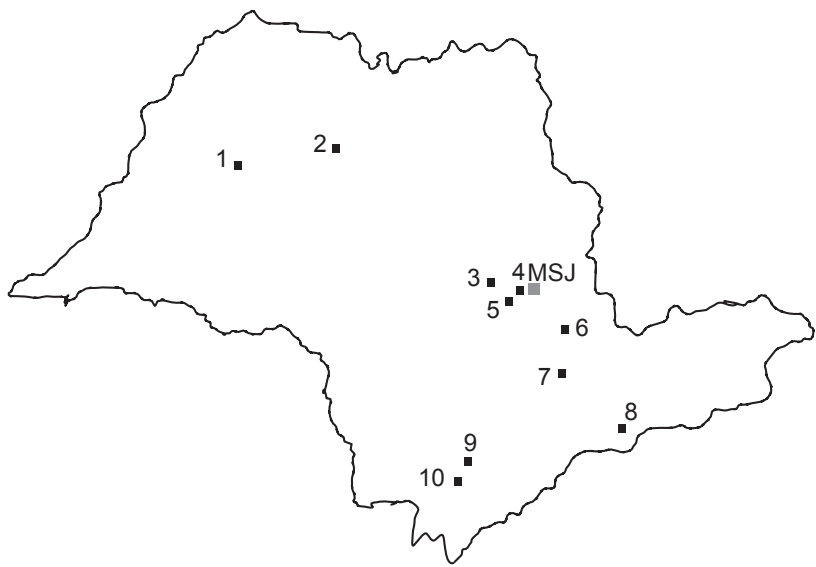

Figura 1. Áreas do Estado de São Paulo comparadas em relação a composição das taxocenoses de anuros: 1) Município de Guararapes; 2) Distrito de Nova Itapirema (Município de Nova Aliança); 3) Estação Ecológica de Itirapina (Município de Itirapina); 4) Floresta Estadual Edmundo Navarro de Andrade (Município de Rio Claro); 5) Distrito de Itapé (Município de Rio Claro); 6) Mata de Santa Genebra (Município de Campinas); 7) Serra do Japi (Município de Jundiaí); 8) Reserva Biológica de Boracéia (Município de Boracéia); 9) Parque Estadual de Intervales (Município de Ribeirão Grande); e 10) Município de Ribeirão Branco. Em destaque a área em que o presente estudo foi realizado; Mata São José (MSJ).

Figure 1. Areas in the state of São Paulo in which the anuran species composition were compared: 1) Municipality of Guararapes; 2) Nova Itapirema (municipality of Nova Aliança); 3) Estação Ecológica de Itirapina (mnicipality of Itirapina); 4) Floresta Estadual Edmundo Navarro de Andrade (municipality of Rio Claro); 5) Itapé (municipality of Rio Claro); 6) Mata Santa Genebra (municipality of Campinas); 7) Serra do Japi (municipality of Jundiaí); 8) Reserva Biológica de Boracéia (municipality of Boracéia); 9) Parque Estadual de Intervales (municipality of Ribeirão Grande); and 10) Municipality of Ribeirão Branco. The studied area (Mata São José: MSJ) is in evidence in the picture.

ro: originalmente uma área de floresta semidecídua, atualmente é uma floresta de eucaliptos com sub-bosque em regeneração. O levantamento da anurofauna da área foi realizado entre 2001 e 2002 por Toledo et al. (2003), quando foram visitados os seguintes ambientes: campos abertos com formação de alagados, lagoa permanente, córrego (os três localizados em áreas fortemente antropizadas), lagoa permanente em mata secundária e córrego em área de mata primária. A Floresta Edmundo Navarro de Andrade dista cerca de $5 \mathrm{~km}$ da MSJ com a qual possui conexão através de matas de galeria do Ribeirão Claro;

5) Distrito de Itapé ( $22^{\circ} 24^{\prime} \mathrm{S}$ e $47^{\circ} 33^{\prime} \mathrm{W}$; $635 \mathrm{~m}$ de altitude), município de Rio Claro: área totalmente degradada, constituindo-se basicamente por ambientes de pastagens. A anurofauna foi inventariada de 1995 a dezembro de 2005 (C.F.B. Haddad \& L.F. Toledo, dados não publicados) (Tabela 1), em ambientes temporários (poças e lagoas) e lagoas permanentes localizados em áreas perturbadas. Dista cerca de $15 \mathrm{~km}$ da MSJ;

6) Mata de Santa Genebra (MSG: $22^{\circ} 44^{\prime} \mathrm{S}$ e $47^{\circ} 06^{\prime} \mathrm{W} ; 670 \mathrm{~m}$ de altitude), município de Campinas: possui composição vegetal semelhante a encontrada no fragmento presentemente estudado, no entanto seu estado de preservação é um pouco melhor que o da MSJ. A lista de anuros do local foi fornecida por I. Sazima (dados não publicados; Tabela 1) com base em coletas realizadas entre 1985 e 1995 em ambientes permanentes no entorno do fragmento (lagoa, região brejosa e córrego) e em locais no interior do fragmento (trilhas). Dista cerca de 80 km da MSJ;

7) Serra do Japi ( $23^{\circ} 11^{\prime} \mathrm{S}$ e $46^{\circ} 52^{\prime} \mathrm{W}$; $900 \mathrm{~m}$ de altitude), município de Jundiaí: área tida como um ecótono de floresta estacional semidecídua e floresta ombrófila densa. O primeiro levantamento desta área foi realizado entre os anos de $1988 \mathrm{e}$ 1999 (Haddad \& Sazima 1992) em um açude permanente de aproximadamente $100 \mathrm{~m}$ de diâmetro, e em córregos e poças temporárias adjacentes ao açude. $\mathrm{O}$ açude localiza-se na chamada Serra da Erminda que corresponde a um segmento da Serra do Japi. Recentemente algumas espécies foram acrescentadas à lista por Ribeiro et al. (2005) com dados coletados entre 2002 e 2005 na mesma área inventariada por Haddad \& Sazima (1992) e em lagoas permanentes, poças temporárias e córregos no interior da Reserva Municipal da Serrra do Japi. A Serra do Japi dista cerca de $120 \mathrm{~km}$ da MSJ;

8) Reserva Biológica de Boracéia (RBB: $23^{\circ} 38^{\prime} \mathrm{S}$ e $45^{\circ} 52^{\prime} \mathrm{W}$; $900 \mathrm{~m}$ de altitude), município de Boracéia: é uma área preservada de floresta ombrófila densa. O levantamento da anurofauna foi realizado por Heyer et al. (1990) com base em dados coletados por diferentes pesquisadores entre o final dos anos 40 e início dos anos 80, quando foram amostrados os seguintes ambientes: córregos e poças temporárias no interior de mata e uma lagoa em ambiente aberto e antropizado. A Reserva Biológica de Boracéia dista cerca de $140 \mathrm{~km}$ da MSJ;

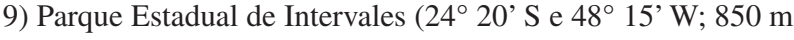
de altitude), município de Ribeirão Grande: uma área conservada de floresta ombrófila densa na Serra de Paranapiacaba. O levantamento da anurofauna foi realizado com base em dados coletados entre 1990 e 1991 (Bertoluci 1998, Bertoluci \& Rodrigues 2002) nos seguintes ambientes: três lagoas permanentes localizadas no interior da mata, na interface da mata primária e área aberta e na borda de floresta secundária, charco localizado em área aberta, área encharcada com uma lagoa semipermanente central e margens de um lago permanente em área sob forte influência antrópica. O Parque Estadual de Intervales dista cerca de $220 \mathrm{~km}$ da MSJ; e

10) Município de Ribeirão Branco (24 $13^{\prime}$ S e $48^{\circ} 46^{\prime} \mathrm{W}$; 800 m de altitude): originalmente era uma área de ecótono de floresta estacional semidecídua e floresta ombrófila densa com elementos de floresta de araucária. Sua anurofauna foi levantada entre 1993 e 2004 por Pombal \& Haddad (2005), quando foi visitada uma poça permanente, localizada na divisa do município de Ribeirão Branco e Apiaí, em área alterada por atividade agropecuária. Dista cerca de $240 \mathrm{~km}$ da MSJ.

O grau de similaridade das taxocenoses da MSJ e das localidades acima relacionadas foi determinado pela aplicação do coeficiente de Jaccard (Cj) (Pielou 1984), com posterior análise de agrupamento pelo método de Cluster Average. Para estas análises e para a confecção do dendrograma foi utilizado o programa BioDiversity Pro 2. Para verificar a existência de relação entre a composição da anurofauna dos diferentes locais analisados com a distância deles à MSJ foi realizada uma análise de regressão entre os índices de similaridade, sendo considerados significativos valores de $\mathrm{P}<0,05$ (Zar 1999).

\section{Resultados}

\section{Ocorrência sazonal e modos reprodutivos}

Foram registradas 24 espécies de anuros distribuídas em cinco famílias (Tabela 1): Bufonidae (2 espécies), Cycloramphidae (1 espécie),Hylidae(11 espécies),Leptodactylidae(4especies), Leiuperidae (4 especies) e Microhylidae (2 espécies). Quatorze espécies foram encon- 
Tabela 1. Listas das espécies de anfíbios anuros da Mata São José (MSJ), Município de Rio Claro, do Distrito de Itapé, Município de Rio Claro e da Mata de Santa Genebra (MSG), Município de Campinas, todas no Estado de São Paulo.

Table 1. Anurans of Mata São José (MSJ), mucicipality of Rio Claro, District of Itapé, municipality of Rio Claro and Mata Santa Genebra (MSG), municipality of Campinas. All the municipalities are in the state of Sao Paulo.

\begin{tabular}{|c|c|c|c|}
\hline \multirow[t]{3}{*}{ Família / Espécie } & \multicolumn{3}{|c|}{ Localidade (número de espécies) } \\
\hline & MSJ (24) & Itapé (29) & MSG (17) \\
\hline & Presente estudo & $\begin{array}{c}\text { C.F.B. Haddad \& } \\
\text { L. F. Toledo, dados não } \\
\text { publicados }\end{array}$ & $\begin{array}{c}\text { I. Sazima, } \\
\text { dados não publicados }\end{array}$ \\
\hline \multicolumn{4}{|l|}{ BUFONIDAE } \\
\hline Chaunus ictericus & & & $\mathrm{x}$ \\
\hline C. ornatus & $\mathrm{x}$ & $\mathrm{x}$ & \\
\hline C. schneideri & $\mathrm{x}$ & $\mathrm{x}$ & $\mathrm{x}$ \\
\hline \multicolumn{4}{|l|}{ CYCLORAMPHIDAE } \\
\hline Proceratophrys boiei & $\mathrm{x}$ & & $\mathrm{x}$ \\
\hline \multicolumn{4}{|l|}{ HYLIDAE } \\
\hline Dendropsophus elianeae & $\mathrm{x}$ & $\mathrm{x}$ & \\
\hline D. minutus & $\mathrm{x}$ & $\mathrm{x}$ & $\mathrm{x}$ \\
\hline D. nanus & $\mathrm{x}$ & $\mathrm{x}$ & $\mathrm{x}$ \\
\hline D. sanborni & $\mathrm{x}$ & $\mathrm{x}$ & $\mathrm{x}$ \\
\hline Hypsiboas albopunctatus & $\mathrm{x}$ & $\mathrm{x}$ & \\
\hline H. faber & $\mathrm{x}$ & $\mathrm{x}$ & \\
\hline H.lundii & $\mathrm{x}$ & $\mathrm{x}$ & \\
\hline H. prasinus & $\mathrm{x}$ & $\mathrm{x}$ & $\mathrm{x}$ \\
\hline Itapotihyla langsdorffii & & $\mathrm{x}$ & \\
\hline Phyllomedusa burmeisteri & & & $\mathrm{x}$ \\
\hline Pseudis paradoxa & & & $\mathrm{x}$ \\
\hline Scinax cf. berthae & & $\mathrm{x}$ & \\
\hline S. fuscomarginatus & $\mathrm{x}^{*}$ & $\mathrm{x}$ & $\mathrm{x}$ \\
\hline S. fuscovarius & $\mathrm{x}$ & $\mathrm{x}$ & $\mathrm{x}$ \\
\hline S. similis & $\mathrm{x}$ & $\mathrm{x}$ & \\
\hline Trachycephalus venulosus & & $\mathrm{x}$ & \\
\hline \multicolumn{4}{|l|}{ LEPTODACTYLIDAE } \\
\hline Leptodactylus furnarius & & $\mathrm{x}$ & \\
\hline L. fuscus & $\mathrm{x}$ & $\mathrm{x}$ & $\mathrm{x}$ \\
\hline L. labyrinthicus & & $\mathrm{x}$ & $\mathrm{x}$ \\
\hline L. mystaceus & $\mathrm{x}$ & $\mathrm{x}$ & $\mathrm{x}$ \\
\hline L. mystacinus & $\mathrm{x}$ & $\mathrm{x}$ & $\mathrm{x}$ \\
\hline Leptodactylus cf. ocellatus & $\mathrm{x}$ & $\mathrm{x}$ & \\
\hline \multicolumn{4}{|l|}{ LEIUPERIDAE } \\
\hline Eupemphix nattereri & $\mathrm{x}$ & $\mathrm{x}$ & \\
\hline Physalaemus centralis & $\mathrm{x}$ & $\mathrm{x}$ & \\
\hline Physalaemus cuvieri & $\mathrm{x}$ & $\mathrm{x}$ & $\mathrm{x}$ \\
\hline Physalaemus fuscomaculatus & $\mathrm{x}$ & $\mathrm{x}$ & \\
\hline Pseudopaludicola cf. falcipes & & $\mathrm{x}$ & \\
\hline Pseudopaludicola sp. (aff. saltica) & & $\mathrm{x}$ & \\
\hline \multicolumn{4}{|l|}{ MICROHYLIDAE } \\
\hline Chiasmocleis albopunctata & $\mathrm{x}$ & & \\
\hline Elachistocleis cf. ovalis & $\mathrm{x}$ & $\mathrm{x}$ & $\mathrm{x}$ \\
\hline
\end{tabular}

*espécie encontrada após o período do estudo

tradas em atividade de vocalização durante três ou mais meses consecutivos (Tabela 2). Cinco espécies, Dendropsophus elianeae, Scinax similis, Leptodactylus mystaceus, Proceratophrys boiei e Chaunus ornatus, foram encontradas em atividade de vocalização por no máximo dois meses seqüenciais. Leptodactylus cf. ocellatus, Physalaemus centralis e $P$. marmoratus foram observadas em atividade de vocalização apenas após dias chuvosos (Tabela 2). Chaunus ornatus, Hypsiboas lundii e
Proceratophrys boiei interromperam a atividade de vocalização por períodos de um a seis meses retomando-a depois. Todas as espécies foram exclusivamente noturnas e apenas Chiasmocleis albopunctata foi registrada somente pela presença de girinos em estágio inicial de desenvolvimento. Scinax fucomarginatus foi encontrada em atividade de vocalização após o término do período de coletas, não sendo possível determinar seu período reprodutivo. 
Tabela 2. Ocorrência temporal das espécies de anuros da Mata São José (fragmento e seu entorno) entre agosto de 2002 e julho de 2003 (Scinax fuscomarginatus foi registrada em 2004 durante uma visita esporádica), Município de Rio Claro, Estado de São Paulo, e respectivos modos reprodutivos segundo Haddad \& Prado (2005).

Table 2. Temporal occurrences of the anurans of Mata São José (including its surrounding areas), municipality of Rio Claro, state of Sao Paulo and reproductive modes of the species (sensu Haddad \& Prado, 2005). Data collected between August/2002 and July/2003. Scinax fucomarginatus was registered in 2004 during a sporadic field trip.

\begin{tabular}{|c|c|c|c|c|c|c|c|c|c|c|c|c|c|}
\hline \multirow[t]{2}{*}{ Espécies } & \multirow{2}{*}{$\begin{array}{c}\text { Modo } \\
\text { Reprodutivo }\end{array}$} & \multicolumn{12}{|c|}{ Meses de Estudo } \\
\hline & & ago & set & out & nov & dez & jan & fev & mar & abr & mai & jun & jul \\
\hline \multicolumn{14}{|l|}{ BUFONIDAE } \\
\hline Chaunus ornatus & 1 & & $\bullet$ & & & & & & & $\bullet$ & - & & - \\
\hline C. schneideri & 1 & & & $\bullet$ & $\bullet$ & - & & & & & & & \\
\hline \multicolumn{14}{|l|}{ CYCLORAMPHIDAE } \\
\hline Proceratophrys boiei & 1 & $\bullet$ & & & & $\bullet$ & $\bullet$ & & & & & & \\
\hline \multicolumn{14}{|l|}{ HYLIDAE } \\
\hline Dendropsophus elianeae & 1 & & & & & - & - & & & & & & \\
\hline D. minutus & 1 & & & & - & $\bullet$ & - & $\bullet$ & $\bullet$ & & & & \\
\hline D. nanus & 1 & & $\bullet$ & $\bullet$ & $\bullet$ & $\bullet$ & $\bullet$ & $\bullet$ & & & & & \\
\hline D. sanborni & 1 & & $\bullet$ & $\bullet$ & $\bullet$ & $\bullet$ & $\bullet$ & $\bullet$ & & & & & \\
\hline Hypsiboas albopunctatus & 1 & & $\bullet$ & $\bullet$ & - & $\bullet$ & $\bullet$ & $\bullet$ & $\bullet$ & $\bullet$ & $\bullet$ & & \\
\hline H. faber & 4 & & & & & & - & $\bullet$ & - & & & & \\
\hline H. lundii & 4 & & $\bullet$ & $\bullet$ & $\bullet$ & - & & & $\bullet$ & $\bullet$ & & & \\
\hline H. prasinus & 2 & & $\bullet$ & $\bullet$ & - & $\bullet$ & $\bullet$ & $\bullet$ & $\bullet$ & $\bullet$ & $\bullet$ & - & - \\
\hline Scinax fuscovarius & 1 & & & & $\bullet$ & $\bullet$ & $\bullet$ & $\bullet$ & & & & & \\
\hline Scinax similis & 1 & & & & & & & $\bullet$ & - & & & & \\
\hline \multicolumn{14}{|l|}{ LEPTODACTYLIDAE } \\
\hline Leptodactylus fuscus & 30 & & & $\bullet$ & - & $\bullet$ & - & - & & & & & \\
\hline L. mystacinus & 30 & & & $\bullet$ & $\bullet$ & $\bullet$ & & & & & & & \\
\hline L. mystaceus & 30 & & & $\bullet$ & $\bullet$ & & & & & & & & \\
\hline Leptodactylus cf. ocellatus & 11 & & & & $\bullet$ & & & & & & & & \\
\hline \multicolumn{14}{|l|}{ LEIUPERIDAE } \\
\hline Eupemphix. nattereri & 11 & & & $\bullet$ & $\bullet$ & $\bullet$ & $\bullet$ & & & & & & \\
\hline Physalaemus centralis & 11 & & & & - & & & & & & & & \\
\hline P. cuvieri & 11 & & & $\bullet$ & $\bullet$ & $\bullet$ & $\bullet$ & $\bullet$ & $\bullet$ & $\bullet$ & & & \\
\hline P. fuscomaculatus & 11 & & & & $\bullet$ & & & & & & & & \\
\hline \multicolumn{14}{|l|}{ MICROHYLIDAE } \\
\hline Chiasmocleis albopunctata * & 1 & & & & & & & & & & & & \\
\hline Elachistocleis cf. ovalis & 1 & & & & $\bullet$ & - & - & & & & & & \\
\hline
\end{tabular}

* apenas o girino da espécie foi encontrado.

Cinco modos reprodutivos (sensu Haddad \& Prado 2005) estão representados na taxocenose inventariada. O mais comum, apresentado por $52 \%$ das espécies, foi o modo 1 com ovos e girinos exotróficos se desenvolvendo em corpos d'água lêntica. Quatro espécies apresentaram modos reprodutivos mais especializados, tais como as espécies do grupo de Leptodactylus fuscus (L. fuscus, L. mystaceus e $L$. mystacinus), que depositam ovos envoltos em espuma no interior de ninhos subterrâneos construídos pelos machos próximos a corpos de água, com girinos exotróficos que se desenvolvem em poças d'água (Martins 1998) (Tabela 2) e Hypsiboas faber, cujo modo reprodutivo (modo 4 sensu Haddad \& Prado 2005) consiste na deposição de ovos em "panelas" naturais ou construídas pelos machos próximos a corpos d'água, com girinos exotróficos que se desenvolvem nos corpos d'água (Martins \& Haddad, 1998) (Tabela 2).

O número de espécies em atividade de vocalização foi positivamente correlacionado com a pluviosidade mensal $\left(\mathrm{r}^{2}=0,59\right.$; $\mathrm{P}<0,05 ; \mathrm{n}=12$ ) (Figura 2a) e com a temperatura média mensal do ar $\left(\mathrm{r}^{2}=0,56 ; \mathrm{P}<0,05 ; \mathrm{n}=12\right)$ (Figura 2b).

\section{Utilização dos corpos d'água para atividade de vocalização}

A ocorrência e o sítio de vocalização das espécies registradas estão apresentados abaixo, para cada corpo d'água amostrado:

1) Córrego no interior do fragmento: $H$. lundii e H. prasinus, sendo que a primeira ocupou estratos mais altos da vegetação ciliar; 

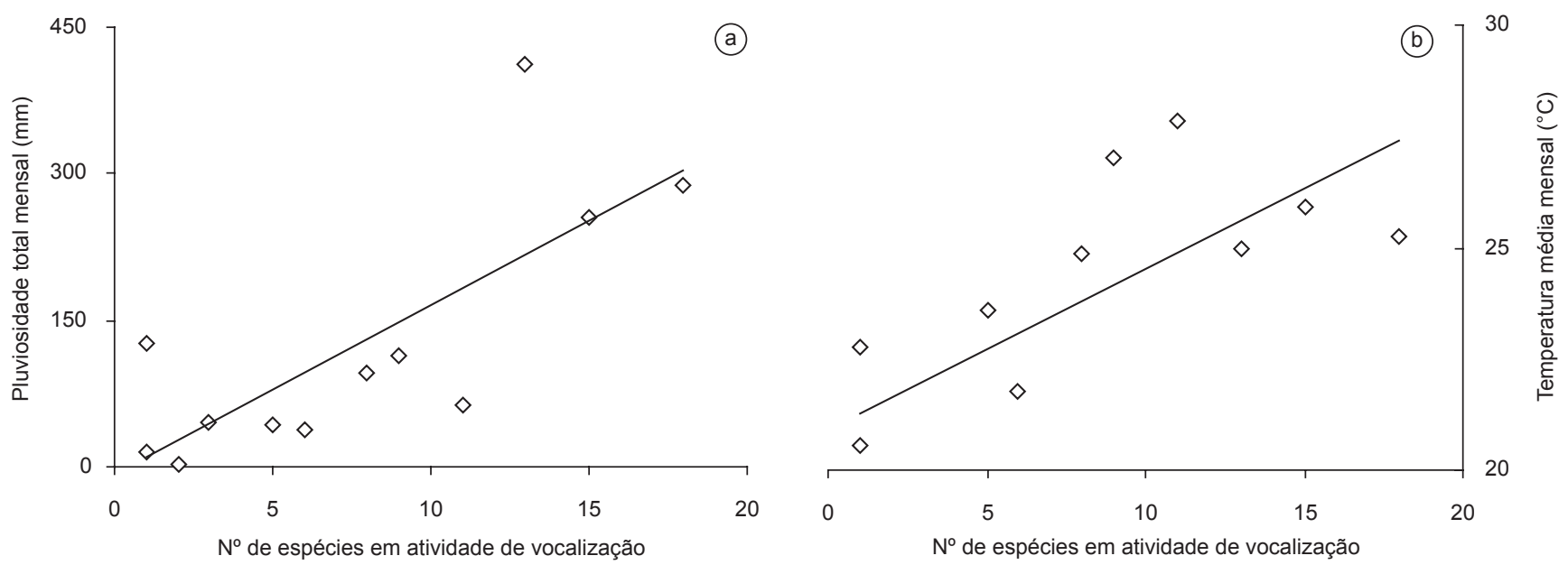

Figura 2. Regressão entre o número de espécies encontradas em atividade de vocalização a) a temperatura média mensal e b) pluviosidade mensal total durante o período de estudo na Mata São José, Município de Rio Claro, Estado de São Paulo.

Figure 2. Linear regression between the number of species found in calling activity and a) the mean air temperature and b) total monthly rainfall during the study period in Mata São José, municipality of Rio Claro, state of São Paulo.

2) Poças temporárias no interior da mata: Proceratophrys boiei, cujos machos vocalizaram nas margens das poças, ora na serapilheira ora imersos na água, e $H$. lundii, cujos machos vocalizaram nos estratos mais altos da vegetação arbórea próximos às poças;

3) Lagoa permanente em área aberta: machos de nove espécies vocalizaram nesse corpo d'água. Três deles vocalizaram nas gramíneas às margens da lagoa: Dendropsophus minutus, D. nanus e D. sanborni. Machos de duas espécies, Hypsiboas albopunctatus e $H$. prasinus vocalizaram em folhas de taboa no interior da lagoa. Apenas os machos de $H$. faber utilizaram estratos vegetacionais mais altos, tais como árvores e arbustos às margens da lagoa. Três espécies vocalizaram parcialmente submersas na água ou sobre solo encharcado: Chaunus ornatus, C. schneideri e Physalaemus cuvieri; e

4) Poças temporárias em área aberta: foram utilizadas por 15 espécies. Machos de seis espécies vocalizaram em gramíneas nas margens desses corpos d'água: Dendropsophus elianeae, D. minutus, D. nanus, D. sanborni, Scinax fuscovarius e $S$. similis. Machos de Eupemphix nattereri, Leptodactylus cf. ocellatus, Physalaemus centralis, P. cuvieri, P. marmoratus e Elachistocleis cf. ovalis vocalizaram imersos na água ou sobre o solo encharcado próximo às poças, enquanto Leptodactylus fuscus, L. mystaceus e L. mystacinus vocalizaram próximos às poças, mas sobre solo seco.

Cerca de $90 \%$ das espécies registradas vocalizaram nos corpos d'água nas áreas adjacentes ao fragmento de floresta amostrado. Apenas machos de Hypsiboas prasinus foram observados em atividade de vocalização em corpos d'água tanto no interior quanto em áreas adjacentes ao fragmento, e os de Proceratophrys boiei e H. lundii, por sua vez, apenas nos corpos d'água no interior do fragmento.

\section{Comparação com outras taxocenoses}

A taxocenose de anuros da MSJ e áreas adjacentes está fortemente relacionada à da FEENA $(\mathrm{Cj}=73,1 \%)$, floresta com a qual está conectada através de uma mata de galeria. A próxima área com maior similaridade com à MSJ é Itapé $(\mathrm{Cj}=67,7 \%)$, seguida da EEI $(\mathrm{Cj}=57,6 \%)$. Estas áreas estão relacionadas com as áreas degradadas do oeste paulista: Nova Aliança $(\mathrm{Cj}=50,0 \%)$ e Guararapes
( $\mathrm{Cj}=39,4 \%)$. Em seguida, aparece a MSG (IS = 48,3\%) fechando um grande agrupamento que reúne as áreas degradadas, Cerrado e florestas semideciduas. O segundo grande agrupamento é constituído pelos ecótonos da Serra do Japi $(\mathrm{Cj}=23,3 \%)$ e Ribeirão Branco $(\mathrm{Cj}=25,9 \%)$ e por áreas de florestas ombrófilas: P. E. Intervales e R. B. Boracéia (Cjs $=25$ e 12,8\%, respectivamente) (Figura 3). A distância entre as localidades comparadas e a MSJ não influenciou o grau de similaridade $\left(\mathrm{r}^{2}=0,17 ; \mathrm{P}=0,13 ; \mathrm{n}=10\right)$; ou seja, as áreas mais próximas da MSJ não são necessariamente as mais similares (Figura 4).

\section{Discussão}

\section{Ocorrência sazonal e modos reprodutivos}

Aproximadamente $61 \%$ das espécies amostradas apresentaram padrão reprodutivo prolongado (sensu Wells 1977) e 74\% responderam positivamente à variação climática de temperatura e precipitação pluviométrica. Este é o padrão que tem sido registrado em regiões subtropicais/sazonais (e.g., Gascon 1991, Rossa-Feres \& Jim 1994, Bertoluci 1998, Toledo et al. 2003). Algumas espécies apresentaram o período reprodutivo bem delimitado temporalmente, enquanto outras entraram em atividade de vocalização mais de uma vez ao longo do ano, tal como ocorreu com Chaunus ornatus, Hypsiboas lundii e Proceratophrys boiei. Segundo Morin (1987), não é claro o porquê algumas espécies são mais rígidas quanto ao seu período reprodutivo enquanto outras se mostram mais flexíveis. Bertoluci \& Rodrigues (2002) também observaram que Chaunus ornatus (referido como Bufo crucifer) apresentou dois períodos reprodutivos distintos. De acordo com estes autores, isto pode representar uma estratégia para a redução da competição intra e interespecífica entre girinos, através da partilha temporal de recursos. No entanto, considerando o modo reprodutivo mais especializado de $H$. lundii e o sítio de reprodução de $P$. boiei (única espécie a utilizar poças temporárias no interior do fragmento), a competição entre girinos parece não explicar o porquê estas espécies interromperam suas atividades reprodutivas retomando-a meses depois. Apesar das espécies citadas terem interrompido a atividade de vocalização em períodos distintos, em ambas este fenômeno pode estar relacionado com 


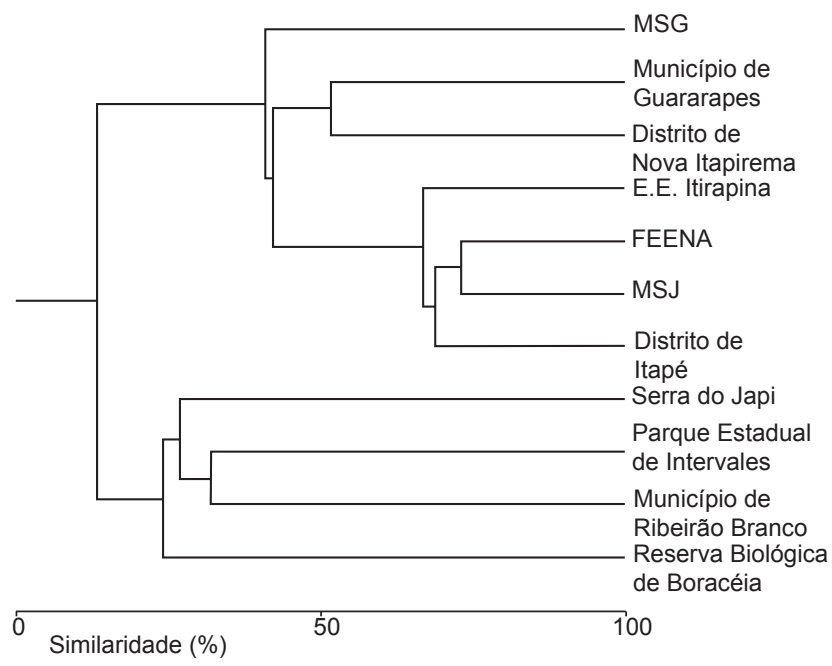

Figura 3. Dendrograma baseado no índice de similaridade de Jaccard, comparando a composição das taxocenoses de anuros de 10 localidades do Estado de São Paulo com a registrada na Mata São José, Município de Rio Claro, Estado de São Paulo.

Figure 3. Dendrogram constructed based on Jaccard similarity index comparing the anuran species composition of 10 areas in the state of São Paulo with those registered in Mata São José, municipality of Rio Claro, state of São Paulo.

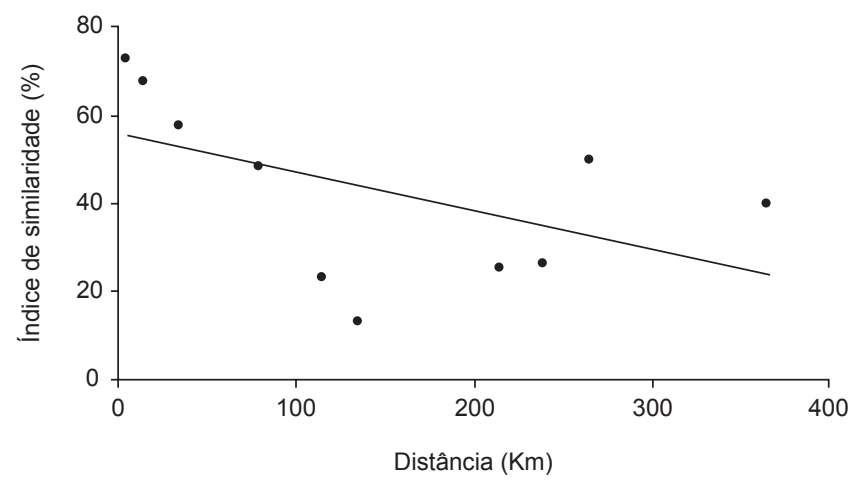

Figura 4. Regressão entre o índice de similaridade Jaccard e a distância $(\mathrm{km})$ entre a Mata São José e as 10 localidades do Estado de São Paulo, cujas taxocenoses foram comparadas com a da Mata São José, Município de Rio Claro, Estado de São Paulo.

Figure 4. Linear regression between the Jaccard similarity index and the distance $(\mathrm{km})$ among MSJ and the 10 areas in the state of São Paulo, which species composition was compared with those of Mata São José, municipality of Rio Claro, state of São Paulo.

fatores abióticos como aumento da precipitação e/ou diminuição da temperatura do ar.

A maioria das espécies amostradas, cerca de $57 \%$, apresentou o modo reprodutivo 1 (Haddad \& Prado 2005), considerado o mais generalizado entre os anuros (Haddad \& Prado 2005), sendo que grande parte destas espécies foram encontradas em corpos de água permanente no entorno do fragmento. $\mathrm{O}$ segundo modo reprodutivo mais frequiente observado no presente estudo foi a desova em ninhos de espuma (modos reprodutivos 11 e 30, sensu Haddad \& Prado
2005), observado em cerca de $35 \%$ das espécies. O fato que todas as espécies que apresentaram estes dois modos reprodutivos foram encontradas em ambientes temporários no entorno do fragmento, corrobora a indicação de que essas espécies teriam maior facilidade em colonizar áreas abertas e ambientes sazonais (Duellman, 1985).

\section{Utilização dos corpos d'água para atividade de vocalização}

A relação espécie/microhábitat é, em geral, maior em ambientes abertos que em florestados, devido ao pequeno número de microhábitats encontrados no primeiro (Cardoso et al. 1989). No presente estudo esta razão foi substancialmente maior no entorno do fragmento; apenas três espécies utilizaram o interior da mata para a reprodução. Isto pode ser consequiência dos desmatamentos ocorridos na área, o que pode ter causado tanto a perda de microhábitats e conseqüente desaparecimento de espécies de anuros, quanto propiciado a invasão de espécies típicas de áreas abertas (como as de Cerrado) nos locais desflorestados. Hypsiboas lundii e Proceratophrys boiei foram encontradas apenas no interior do fragmento, fato este observado para ambas as espécies também em outras localidades do Estado (para Hypsiboas lundii ver Brasileiro et al. 2005 e para Proceratophrys boiei ver Haddad \& Sazima 1992). Isto demonstra uma forte dependência destas duas espécies por ambientes florestados. Sabe-se que uma das principais causas da diminuição do número de espécies de anuros é a diminuição da cobertura vegetal (revisão em Collins \& Storfer 2003) e que algumas espécies de anuros são favorecidas nestas situações, ampliando suas distribuições geográficas (Haddad \& Sazima 1992). Além disso, a fragmentação dos hábitats aumenta a vulnerabilidade dos fragmentos à invasão de espécies exóticas e espécies nativas ruderais (Paton 1994).

A estrutura da comunidade e a coexistência de espécies que utilizam o mesmo ambiente de reprodução têm sido explicadas pela partilha de recursos reprodutivos (Cardoso et al. 1989; Eterovick \& Sazima 2000, Toledo et al. 2003). A maior parte das espécies amostradas no presente estudo utilizou ambientes distintos para a reprodução. Essa utilização diferencial dos corpos d'água estudados revela uma seleção de hábitats reprodutivos, que é uma característica dos anuros (Hero 1990, Gascon 1991). No caso de espécies simpátricas, esta utilização diferenciada pode operar como um eficiente mecanismo de isolamento reprodutivo, permitindo a coexistência entre elas num mesmo corpo d'água. A partilha de recursos pode ocorrer em vários níveis distintos, tais como: uso de diferentes ambientes para a reprodução (sítios de canto e oviposição), turnos e temporadas de vocalização distintas, uso de diferentes canais acústicos e diferenças nas dietas (Rossa-Feres \& Jim 1996, 2001). Por outro lado, foi observado que algumas espécies, inclusive taxonomicamente próximas, utilizaram sítios de vocalização semelhantes e apresentaram mesmo período reprodutivo, tais como: Leptodactylus mystacinus, $L$. fuscus e $L$. mystaceus, Dendropsophus nanus e D. sanborni, Physalaemus centralis, P. cuvieri e P. marmoratus.

\section{Comparação com outras taxocenoses}

A alta similaridade na composição da anurofauna entre a MSJ e a FEENA deve-se a proximidade geográfica entre elas e a conexão através de uma mata de galeria. A FEENA é uma área que sofreu bastante modificação ao longo das últimas décadas; originalmente a área era coberta por floresta estacional semidecídua, posteriormente a região foi transformada em fazenda de plantio de café, e após esta modificação foram plantados diversos talhões de Eucaliptus. Atualmente a paisagem da FEENA caracteriza-se por sub-bosque de mata estacional semidecídua em crescimento em meio a plantações de Eucaliptus. A MSJ atualmente poderia estar servindo como fonte 
de espécies para a FEENA, pois apesar de sua área bastante inferior em tamanho, nela ainda ocorre Proceratophrys boiei, uma espécie sensível ao desmatamento e que pode ter habitado a mata original da FEENA, mas, após as constantes interferências antrópicas, pode ter sido extinta localmente. Hypsiboas lundii ainda pode ser encontrada na FEENA (ver Toledo et al. 2003) e deve ter suportado tais alterações habitando as matas de galeria, que foram pouco alteradas.

Em seguida as áreas que apresentaram maior índice de similaridade com a MSJ foram áreas de Cerrado e as áreas nas quais a vegetação original foi totalmente substituída por culturas e pastagens. Isto pode sugerir que ocorreu uma substituição de populações naturais de anuros da MSJ por espécies típicas de áreas abertas devido a sua fragmentação e ao desmatamento no seu entorno. O mesmo pode ser dito para a MSG que possui área similar a da MSJ e comporta taxocenose típica de áreas alteradas, e foi inserida no agrupamento de áreas alteradas e de Cerrado. Utilizando o índice de coeficiente de semelhança Biogeográfica, Bastos et al. (2003) constataram que a taxocenose de anuros do Município de Rio Claro está relacionada com taxocenoses do Cerrado e de áreas degradadas do Brasil central, Minas Gerais e Goiás.

A Serra do Japi aparece no agrupamento das formações vegetais com influência do domínio da Serra do Mar (P. E. Intervales e Ribeirão Branco), fato este que pode ser facilmente observado em alguns trechos da vegetação arbórea da Serra do Japi, que possuem características fisionômicas típicas da Serra do Mar (Leitão-Filho 1992).

Embora o grau de similaridade entre as localidades comparadas não tenha sido influenciado pela distância entre elas, nota-se que as localidades mais próximas agruparam-se mais fortemente, o que pode estar relacionado à similaridade nas formações vegetais.

Por fim, ressaltamos a necessidade de aumentar o número de estudos em remanescentes de florestas estacionais semidecíduas do Estado de São Paulo e do Brasil, que vêm sendo continuamente fragmentados e desmatados, gerando distribuições disjuntas, que podem resultar em declínios populacionais e extinções de espécies (ver Silvano et al. 2003).

\section{Agradecimentos}

Agradecemos a Celso Araújo, Maurício Godoy e Fábio B. Britto pela ajuda nos trabalhos de Campo; Ivan Sazima por conceder dados inéditos sobre a Mata de Santa Genebra; Denise Rossa Feres pela leitura cuidadosa e comentários. Aos dois revisores anônimos pelas valiosas sugestões. À FAPESP, CAPES e CNPq pelos auxílios concedidos a LFT, CFBH e ao Laboratório de Herpetologia.

\section{Referências}

ARZABE, C. 1999. Reproductive activity patterns of anurans in two different altitudinal sites within the Brazilian Caatinga. Rev. Brasil. Zool. 16(1):851-864.

ÁVILA, R.W. \& FERREIRA, V.L. 2004. Riqueza e densidade de vocalizações de anuros (Amphibia) em uma área urbana de Corumbá, Mato Grosso do Sul, Brasil. Rev. Brasil. Zool. 21(4):887-892.

BASTOS, R.P., MOTTA, J.A.O., LIMA, L.P. \& GUIMARÃES, L.D. 2003. Anfíbios da Floresta Nacional de Silvânia, Estado de Goiás. Stylo Gráfica e Editora, Goiânia.

BERNADE, P.S. \& KOKUBUM, M.N.C. 1999. Anurofauna do Município de Guararapes, Estado de São Paulo, Brasil (Amphibia: Anura). Acta Biol. Leopol. 21(1):89-97.

BERNADE, P.S. \& MACHADO, R.A. 2001. Riqueza de espécies, ambientes de reprodução e temporada de vocalização da anurofauna em Três Barras do Paraná, Brasil (Amphibia: Anura). Cuad. Herpetol. 14(2):93-104.

BERTOLUCI, J. 1998. Annual patterns of breeding activity in Atlantic rainforest anurans. J. Herpetol. 32(4):607-611.
BERTOLUCI, J. \& RODRIGUES, M.T. 2001. Utilização de hábitats reprodutivos e micro-hábitats de vocalização em uma taxocenose de anuros (Amphibia) da Mata Atlântica do sudeste do Brasil. Pap. Avul. Zool. 42(11):287-297.

BERTOLUCI, J. \& RODRIGUES, M.T. 2002. Seasonal patterns of breeding activity of Atlantic rainforest anurans at Boracéia, southeastern Brazil. Amphibia-Reptilia 23(2):161-167.

BRASILEIRO, C.A., SAWAYA, R.J., KIEFER, M.C. \& MARTINS, M. 2005. Amphibians of an open Cerrado fragment in southeastern Brazil. Biota Neotropica 5(2): http://www.biotaneotropica.org.br/v5n2/pt/abstract?ar ticle+BN00405022005 (ultimo acesso 12/06/2006).

CARDOSO, A.J., ANDRADE, G.V. \& HADDAD, C.F.B. 1989. Distribuição espacial em comunidades de anfíbios (Anura) no sudeste do Brasil. Rev. Brasil. Biol. 49(1):241-249.

COLLINS, J.P. \& STORFER, A. 2003. Global amphibian declines: sorting the hypotheses. Divers. Distrib. 9(2):89-98.

DUELLMAN, W.E. 1985. Reproductive modes in anuran amphibians: phylogenetic significance of adaptive strategies. S. Afr. J. Sci. 81(4):174-178.

DUELLMAN, W.E. 1999. Distribution patterns of amphibians in South America. In Patters of distribution of amphibians: a global perspective (W.E. Duellman, ed.). The Johns Hopkins Univ. Press., Beltimore and London, p.255-328.

ETEROVICK, P.C. \& SAZIMA, I. 2000. Structure of an anuran community in a montane meadow in southeastern Brazil: effects of seasonality habitats, and predation. Amphibia-Reptilia 21(4):439-461.

FUNK, W.C., GREENE, A.E, CORN, P.S. \& ALLENDORF, F.W. 2005. High dispersal in a frog species suggests that it is vulnerable to habitat fragmentation. Biol. Lett. 1(1):1-4.

GASCON, C. 1991. Population and community level analyses of species occurrences of central Amazonian rainforest tadpoles. Ecology 72(5):1731-1746.

HADDAD, C.F.B. 1998. Biodiversidade dos anfíbios no Estado de São Paulo. In Biodiversidade do Estado de São Paulo: síntese do conhecimento ao final do século XX (R.M.C. Castro, ed.). Editora FAPESP, São Paulo, v.6, p.17-26.

HADDAD, C.F.B. \& PRADO, C.P.A. 2005. Reproductive modes in frogs and their unexpected diversity in the Atlantic forest of Brazil. BioScience 55(3):207-217.

HADDAD, C.F.B. \& SAZIMA, I. 1992. Anfíbios anuros da Serra do Japi. In História natural da Serra do Japi: ecologia e preservação de uma área florestal no sudeste do Brasil (L.C.P Morellato, ed.). Editora da Unicamp/ Fapesp, Campinas, p.188-211.

HERO, J.M. 1990. An Illustrated key to tadpoles occurring in the Central Amazon rainforest, Manaus, Amazonas, Brazil. Amazoniana XI:201-262.

HEYER, W.R., RAND, A.S., CRUZ, C.A.G., PEIXOTO, O.L. \& NELSON, C.E. 1990. Frogs of Boracéia. Arq. Zool. 31(4):231-410.

HITCHINGS, S.P. \& BEEBEE, T.J.C. 1997. Genetic substructuring as a result of barriers to gene flow in urban Rana temporaria (common frog) populations: implications for biodiversity conservation. Heredity 79(2):117-127.

JOHNSTON, B. \& FRID, L. 2002. Clearcut logging restricts the movements of terrestrial Pacific giant salamanders (Dicamptodon tenebrosus). Can. J. Zool. 80(12):2170-2177.

KATS, L.B. \& FERRER, R.P. 2003. Alien predators and amphibian declines: review of two decades of science and transition to conservation. Diver. Distrib. 9(2):99-110.

LEITÃO-FILHO, H.F. 1992. A flora arbórea da Serra do Japi. In História natural da Serra do Japi: ecologia e preservação de uma área florestal no sudeste do Brasil (L.P.C. Morellato, ed.). Editora da Unicamp/Fapesp, Campinas, p.40-62.

MARTINS, M. 1998. Biologia reprodutiva de Leptodactylus fuscus em Boa Vista, Roraima (Amphibia: Anura). Rev. Bras. Biol. 48(4):969-977.

http://www.biotaneotropica.org.br 
MARTINS, M. \& HADDAD, C.F.B. 1998. Vocalizations and reproductive behaviour in the Smith frog, Hyla faber Weed (Amphibia: Hylidae). Amphibia-Reptilia 9:49-60.

MORIN, P.J. 1987. Predation, breeding asynchrony, and the outcome of competition among tree frog tadpoles. Ecology 68(3):675-683.

PATON, P.W.C. 1994. The effect of edge on avian nest success: how strong is the evidence? Conserv. Biol. 8(1):17-26.

PIELOU, E.C. 1984. The interpretation of Ecological Data. Wiley, New York.

POMBAL, JR.J.P. \& HADDAD, C.F.B. 2005. Estratégias e modos reprodutivos de anuros (Amphibia) em uma poça permanente na Serra de Paranapiacaba, Sudeste do Brasil. Pap. Avul. Zool 45(15):201-213.

RIBEIRO, R.S.; EGITO, G.T.B.T. \& HADDAD, C.F.B. 2005. Chave de identificação: anfíbios anuros da vertente de Jundiaí da Serra do Japi, Estado de São Paulo. Biota Neotropica 5(2): http://www.biotaneotropica. org.br/v5n2/pt/abstract?identification-key+bn03005022005. ISSN 16760603 (ultimo acesso 12/06/2006).

ROSSA-FERES, D.C. \& JIM, J. 1994. Distribuição sazonal em comunidades de anfíbios anuros na região neotropical de Botucatu, São Paulo. Rev. Brasil. Biol 54(2):323-334.

ROSSA-FERES, D.C. \& JIM, J. 1996. Distribuição espacial em comunidades de girinos na região de Botucatu, São Paulo (Amphibia: Anura). Rev. Brasil. Biol. 56(2):309-316.
ROSSA-FERES, D.C. \& JIM, J. 2001. Similaridade do sítio de vocalização em uma comunidade de anfíbios anuros na região noroeste do Estado de São Paulo, Brasil. Rev. Brasil. Biol. 18(2):439-454.

SILVANO, D.L., COLli, G.R., DIXOM, M.B.O., PIMENTA, B.V.S. \& WIEDERHECKER, H.C. 2003. Fragmentação de Ecossistemas: causas, efeitos sobre a biodiversidade e recomendações de políticas públicas. Ministério do Meio Ambiente, Brasília.

TOLEDO, L.F., ZINA, J. \& HADDAD, C.F.B. 2003. Distribuição espacial e temporal de uma comunidade de anfíbios do Município de Rio Claro, São Paulo, Brazil. Holos Environ. 3(2):136-149.

VELOSO, H.P. \& GÓES-FILHO, L. 1982. Fitogeografia Brasileira: classificação fisionômico-ecológica da vegetação neotropical. Boletim técnico do Projeto RADAMBRASIL, série vegetação, 1:1-80.

VIANA, V.M. \& TABANEZ, A.A.J. 1996. Biology and conservation of forest fragments in the Brazilian Atlantic Moist Forest. In Forest patches in tropical landscapes (R. Schellas \& R. Greenberg, eds.). Island Press., Washington, p.151-167.

WELLS, K.D. 1977. The social behaviour of anuran amphibians. Anim. Behav. 25:666-693.

YOUNG, B.E., LIPS, K.R., REASER, J.K., IBANES, R., SALAS, A.W., CEDEÑO, J.R., COLOMA, L.A., RON, S., MARCA, E., MEYER, J.R., MUÑOZ, A., BOLAÑOS, F., CHAVES, G. \& ROMOS, D. 2000. Population declines and priorities for amphibians conservation in Latin America. Conserv. Biol. 15(5):1213-1223.

ZAR, J. 1999. Biostatiscal analysis. Upper Saddle River Prentice Hall, New Jersey. 